\title{
Consensus mondial sur la responsabilité sociale des facultés de médecine
}

\section{Contribution collective de la conférence pour le consensus mondial sur la responsabilité sociale des facultés de medecine ${ }^{1}$}

\section{Global consensus for social accountability of medical schools}

\begin{abstract}
${ }^{1}$ La version princeps de ce texte a été rédigée à l'issue de la conférence pour le consensus mondial sur la responsabilité sociale des facultés de médecine, qui s'est tenue à East London (Afrique du Sud) du 10 au 13 octobre 2010. Ce projet a été développé sous l'égide d'un comité de pilotage international composé des membres suivants : Magdalena Awases (Human Resources for Health (HRH), World health organization - WHO - Regional Office for Africa), Rebecca Bailey (HRH, WHO), Charles Boelen, co-président (consultant international, ancien coordonnateur du programme de l'Organisation mondiale de la santé - OMS - des ressources humaines pour la santé), Mario Dal Poz ( $H R H, W H O)$ Moses Galukande (Makerere University and International Medical Group), Jorge Eduardo Gutiérrez Calivá (Asociación Castarricense de Facultades y Escuelas de Medicina - ACOFEMED), Dan Hunt (Liaison Committee on Medical Education - LCME), Jehu Iputo (Walter Sisulu University), Yusuf Irawan (Hasanuddin University), Ahmed Alkafaji (Jordan University of Sciences and Technology), Joël Ladner (Université de Rouen), Sam Leinster (University of East Anglia), Stefan Lindgren (World Federation for Medical Education - WFME - and Lund University), Khaya Mfenyana (Walter Sisulu University), Tewfik Nawar (Université de Sherbrooke), André-Jacques Neusy (Training for Health Equity Network - THEnet), Björg Pálsdóttir (THEnet), Jean Rochon (Université Laval), Robert Woollard, co-président (University of British Columbia)

La présente traduction française a été élaborée par les membres du groupe «Santé et société » de la Société internationale francophone d'éducation médicale (SIFEM) : Charles Boelen, consultant international, ancien coordonnateur du programme de l'OMS des ressources humaines pour la santé (France), José Gomes, Département de médecine générale, Faculté de médecine et pharmacie, Université de Poitiers (France), Joël Ladner, Département d'épidémiologie et de santé publique, Faculté de Médecine de Rouen, Université de Rouen (France), Luce Pélissier-Simard, Faculté de médecine et des sciences de la santé, Université de Sherbrooke (Canada), Dominique Pestiaux, Centre académique de médecine générale, Faculté de médecine, Université Catholique de Louvain (Belgique), Tewfik Nawar, professeur émérite, Faculté de médecine, Université de Sherbrooke (Canada) et président du conseil d'évaluation de la Conférence internationale des doyens et des facultés de médecine d'expression française (CIDMEF)
\end{abstract}

Mots clés :

Consensus ;

responsabilité sociale ; faculté de médecine
Résumé - Contexte et problématique : Au début du $\mathrm{XX}^{\mathrm{e}}$ siècle, les facultés de médecine firent face à des défis sans précédent pour faire en sorte que la formation des médecins soit efficace et fondée sur des bases scientifiques, comme en témoigne le rapport Flexner en 1910. En ce début du XXI ${ }^{\mathrm{e}}$ siècle, d'autres défis importants sont à relever, notamment : l'amélioration de la qualité, de l'équité, de la pertinence et de l'efficience dans la prestation des services de santé ; l'harmonisation avec les réalités sociétales ; la définition de rôles nouveaux pour les professionnels de santé ; la démonstration de l'impact des interventions sur le niveau de santé des citoyens. Méthodes : Pour tenter de répondre à ces défis, 130 organisations et experts de par le monde, ayant des compétences en éducation médicale, en régulation professionnelle et en politique de santé, participèrent pendant huit mois à une séquence de trois consultations selon la méthode Delphi, couronnée par une conférence de consensus de trois jours modérée par un arbitre extérieur. Résultats : Le consensus comprend dix directions stratégiques pour 
qu'une faculté de médecine soit «socialement responsable», insistant sur des améliorations indispensables pour : répondre aux besoins et défis actuels et futurs de la société ; prioriser en conséquence l'orientation en matière d'éducation, recherche et service ; renforcer la gouvernance et le partenariat avec d'autres acteurs de santé ; évaluer et accréditer en vue de mesurer et d'améliorer la performance et l'impact. Une synergie entre réseaux et organisations est recommandée au niveau mondial pour la mise en œuvre du consensus, avec comme tâches : un plaidoyer pour la reconnaissance de la pertinence du consensus mondial; des consultations pour l'adaptation et l'application du consensus dans différents contextes; une recherche pour la confection de normes illustrant la responsabilité sociale ; une coordination mondiale pour partager les expériences et soutenir les initiatives. Conclusion : Un siècle après la publication du rapport Flexner, le consensus mondial sur la responsabilité sociale des facultés de médecine est un jalon important qui marquera le développement de l'éducation médicale dans le monde à l'avenir.

Keywords:

Consensus; social accountability; medical schools
Abstract - Context and issue: The beginning of the 20th century presented medical schools with unprecedented challenges to become more scientific and effective in the creation of physicians. This was captured in the Flexner report of 1910. The 21st Century presents medical schools with a different set of challenges: improving quality, equity, relevance and effectiveness in health care delivery; reducing the mismatch with societal priorities; redefining roles of health professionals; and providing evidence of impact on people's heath status. Methods: To address those challenges 130 organizations and individuals from around the world with responsibility for health education, professional regulation and policy-making participated for eight months in a three-round Delphi process leading to a three-day facilitated consensus development conference. Results: The Consensus consists of ten strategic directions for medical schools to become socially accountable, highlighting required improvements to: respond to current and future health needs and challenges in society; reorient their education, research and service priorities accordingly; strengthen governance and partnerships with other stakeholders; use evaluation and accreditation to assess performance and impact. It recommends synergy among existing networks and organizations to move the consensus into action at global level, with a number of tasks: advocacy to recognize the value of the global consensus; consultancy to adapt and implement it in different contexts; research to design standards reflecting social accountability; global coordination to share experiences and support. Conclusion: A century after Flexner's report, the global consensus on social accountability of medical schools is a charted landmark for future medical education worldwide.

\section{Introduction}

Un siècle après la parution du rapport Flexner sur l'état de l'éducation médicale en Amérique du Nord, le principal défi pour l'éducation des professionnels de santé en ce $\mathrm{XXI}^{\mathrm{e}}$ siècle réside dans la démonstration par les institutions de formation d'une meilleure contribution à l'amélioration de la performance des systèmes de santé et du niveau de santé des citoyens et des populations, non seulement par une adéquation des programmes de formation aux problèmes prioritaires de santé mais aussi par un plus grand engagement à anticiper les besoins en santé et en professionnels de santé du pays et à veiller à ce que les diplômés pratiquent là où sont les plus grands besoins en dispensant les services les plus attendus. Un nouveau paradigme d'excellence pour l'institution académique s'impose, requérant des normes et des mécanismes d'accréditation permettant de promouvoir et d'évaluer sa capacité à améliorer son impact sur la santé.

\section{Méthodes}

Du 10 au 13 octobre 2010, soixante-cinq délégués représentant des facultés de médecine et des agences 
d'accréditation à travers le monde se sont réunis à East London, en Afrique du Sud, pour finaliser le document de consensus mondial sur la responsabilité sociale des facultés de médecine, dont le contenu est présenté ci-après. Ce fut l'aboutissement d'une préparation et d'une interaction étendues sur deux années avec un Groupe international de référence (GIR), composé de 130 représentants d'organisations et personnalités reconnues pour leur expertise en matière d'éducation médicale, d'accréditation et de responsabilité sociale.

Avec l'aide d'un comité de pilotage de 20 experts internationaux, les membres du GIR participèrent à une suite de trois consultations itératives selon la méthode Delphi étendue sur une période de huit mois et se terminant par la conférence de consensus. Dès la première consultation, quarantetrois pages de commentaires et suggestions furent obtenues en réponse aux trois questions ouvertes suivantes :

- Comment les facultés de médecine devraientelles améliorer leur réponse aux futurs défis de santé dans la société ?

- Comment cette disposition devrait-elle être renforcée, y compris par des systèmes d'accréditation, d'auto-évaluation et d'évaluation par les pairs?

- Comment mesurer les progrès faits en la matière?

À la suite des deux consultations suivantes et de la conférence de consensus, facilitée par un expert indépendant, l'analyse des réponses fit émerger des thèmes généraux et un consensus fut obtenu sur dix axes stratégiques. Chaque axe et son contenu sont ainsi le fruit d'un processus de consultation et de négociation démocratiques garantissant le fait que le consensus fut élaboré à partir de l'expertise et de l'expérience des membres du GIR.

Les dix axes stratégiques déterminés par la conférence pour le consensus mondial sur la responsabilité sociale des facultés de médecine sont les suivants :

Axe 1: Anticipation des besoins en santé de la société.
Axe 2: Création de partenariats avec le système de santé et autres acteurs.

Axe 3: Adaptation aux rôles nouveaux des médecins et autres professionnels de la santé

Axe 4 : Éducation basée sur des résultats escomptés.

Axe 5: Instauration d'une gouvernance réactive et responsable.

Axe 6: Redéfinition de normes pour l'éducation, la recherche et la prestation de services.

Axe 7 : Amélioration continue de la qualité en éducation, recherche et prestation de services.

Axe 8: Institutionnalisation de mécanismes d'accréditation.

Axe 9: Adhésion aux principes universels et adaptation au contexte local.

Axe 10: Prise en compte du rôle de la société.

L'objectif général de l'initiative du consensus mondial sur la responsabilité sociale des facultés de médecine est d'obtenir un agrément sur un cadre conceptuel permettant aux facultés de médecine d'avoir une plus grande influence sur la performance du système de santé et sur le niveau de santé des citoyens et des populations. Ce cadre devrait prévoir un corps de normes et des méthodes d'évaluation et d'accréditation, ainsi que des directives pour satisfaire à ces normes.

Pour répondre à cette aspiration, un déroulement en trois phases a été conçu :

\section{Phase I}

Février-octobre 2010

Recueil des contributions des membres du GIR par la méthode Delphi. Chaque consultation est analysée par le comité de pilotage et le compte rendu renvoyé aux membres du GIR pour alimenter la prochaine consultation et parvenir enfin à un consensus

\section{Phase II}

10-13 octobre 2010

Conférence de consensus à East London, Afrique du Sud, avec les représentants des principales organisations engagées de par le monde dans 
l'amélioration de l'éducation médicale. Le consensus obtenu est fondé sur un document provisoire élaboré sur la base de l'étude Delphi des mois précédents.

\section{Phase III}

\section{Après la conférence}

Des collaborations seront engagées et de nouvelles initiatives prises pour mettre en œuvre les recommandations de la conférence par des activités de plaidoyer, de recherche et de publication.

Nous entrons dans la phase III qui exigera des efforts concertés avec un grand nombre d'organisations et de projets. Avec les organisations représentées dans le GIR et d'autres qui se joindront au mouvement, on pourra espérer des progrès vers les améliorations souhaitées.

Le document qui suit représente un consensus clairement exprimé pour une démarche de qualité suivant les dix axes stratégiques. Cette démarche, comprenant le développement de normes et de systèmes d'évaluation et d'accréditation, est toute orientée vers l'amélioration de l'impact sur la santé de la société et des citoyens, au niveau local comme au niveau mondial. Des avancées dans cette direction constitueront une remarquable contribution pour le $\mathrm{XXI}^{\mathrm{e}}$ siècle.

\section{Le document de consensus}

Le consensus sur la responsabilité sociale couvre un champ s'apparentant à une vision systémique, allant de l'identification des besoins de santé à la vérification de la satisfaction de ces besoins par les facultés de médecine. La liste des dix axes stratégiques reflète cette séquence logique, en commençant par une appréhension du contexte social, la définition des défis et besoins de santé et la création de partenariats pour une action efficace (axes 1 et 2). Tenant compte des autres professionnels de santé, le rôle et les compétences du médecin sont évoqués (axe 3), pour servir d'inspiration aux stratégies éducationnelles (axe 4) que le faculté de médecine est appelée à mettre en œuvre, ainsi que des stratégies concordantes en matière de recherche et de prestation de services (axe 5). Des normes seront nécessaires pour permettre à la faculté d'atteindre un haut niveau d'excellence (axes 6 et 7), que l'autorité nationale se devra de reconnaître (axe 8). Alors que le concept de responsabilité sociale a valeur universelle (axe 9), les sociétés locales apprécieront en dernier ressort la pertinence des réalisations (axe 10).

\section{AXE 1. Anticipation des besoins en santé de la société}

1.1 - Le développement des facultés de médecine s'appuie sur des valeurs fondamentales qui devraient se retrouver dans tout système de santé pour répondre aux besoins de la société : qualité, équité, pertinence, utilisation responsable des ressources, esprit d'innovation, partenariat et garantie de pérennité.

1.2 - La faculté de médecine reconnaît les déterminants sociaux de la santé : politiques, épidémiologiques, démographiques, économiques, culturels, et environnementaux. Elle oriente en conséquence ses programmes d'enseignement, de recherche et de prestation de services.

1.3 - La vision et la mission d'une faculté de médecine sont essentiellement inspirées des besoins actuels et futurs de la société, pour développer l'enseignement (formation initiale, spécialisée et formation médicale continue), la recherche (fondamentale et appliquée) et les prestations de services. La faculté de médecine anticipe les changements requis pour aboutir à un système de santé efficient et équitable et à des professionnels de la santé compétents.

AXE 2. Création de partenariats avec le système de santé et d'autres acteurs

2.1 - La faculté s'engage à travailler en étroite collaboration avec des partenaires dans le secteur sanitaire (notamment les gestionnaires et décideurs des politiques de santé, les services de santé, les associations professionnelles, les autres professionnels de la santé, la société civile) et avec d'autres secteurs en vue d'améliorer la performance du système de 
santé et le niveau de santé de la population, au travers de ses missions d'enseignement, recherche et prestation de services.

2.2 - La qualité de ce partenariat est démontrée par la mise en place d'un processus continu de consultation avec les partenaires pour la conception, la mise en œuvre et l'évaluation des programmes d'éducation, de recherche et de prestation de services, dans le but de répondre aux besoins de santé prioritaires. La faculté conseille les autorités locales et nationales dans la définition des politiques et des stratégies visant à établir un système de santé adapté aux besoins sociétaux.

2.3 - La faculté de médecine reconnaît la communauté qu'elle dessert comme partenaire important et participe à la gestion d'un ensemble cohérent de services de santé au sein de cette communauté dans le respect des valeurs fondamentales : qualité, équité, pertinence et efficience. Dans ce cadre, elle met en œuvre et évalue des modèles innovants, intégrant à la fois la santé individuelle et la santé de la population, l'enseignement et la recherche.

2.4 - La faculté considère que les soins de santé primaires constituent la base de tout système de santé, et porte une attention particulière à l'articulation des services de première ligne, avec les services de niveau secondaire et tertiaire, dans le domaine de l'enseignement, la recherche et la prestation des services. Elle établit un équilibre entre les disciplines pour bien répondre aux besoins de santé. Ceci se reflète dans ses programmes d'enseignement, de recherche et de prestation de services.

\section{AXE 3. Adaptation aux rôles nouveaux des médecins et autres professionnels de santé}

3.1 - La faculté de médecine forme des diplômés ayant une gamme de compétences cohérentes avec les besoins des communautés qu'ils servent, le système de santé dans lequel ils travaillent et l'attente des citoyens. Les compétences sont définies en concertation avec les principaux acteurs de santé, y compris les autres professionnels de santé et des secteurs sociaux, considérant comme impératif un partage efficace et une délégation de tâches entre les membres de l'équipe de santé afin d'assurer des soins de qualité, accessibles à tous et efficaces.

3.2 - La faculté de médecine prépare le médecin à un champ de compétences pertinentes par rapport aux valeurs sus-décrites et au concept de professionnalisme reconnu par les organisations compétentes. De telles compétences incluent l'éthique, le travail en équipe, l'adaptation à la diversité culturelle, le leadership et la communication.

3.3 - En accord avec les besoins liés à l'évolution de la société et les ajustements du système de santé, la faculté de médecine produit une diversité de spécialistes de qualité et en nombre suffisant. Une priorité est donnée à l'encouragement des diplômés à s'engager dans les soins de santé primaires.

3.4 - La faculté de médecine reconnaît qu'indépendamment de leur spécialité future, les médecins doivent être clairement engagés dans des activités de santé publique, de façon cohérente avec leurs activités cliniques. Ceci inclut des activités relatives à la promotion de la santé, la prévention des facteurs de risque et la réadaptation pour les patients et l'ensemble de la population. Les diplômés s'investissent davantage dans des activités de plaidoyer et de réforme du système de santé.

\section{AXE 4. Éducation basée sur des résultats escomptés}

4.1 - La faculté de médecine recrute, sélectionne et aide les étudiants en médecine, ceux-ci reflétant la diversité sociale et les groupes défavorisés.

4.2 - L'éventail complet des interventions éducatives, incluant le contenu et la structure du programme, l'attribution des ressources d'apprentissage, les méthodes d'enseignement, l'évaluation de l'étudiant, le développement professionnel des enseignants et les systèmes d'évaluation, est construit pour répondre aux besoins individuels et sociétaux.

4.3 - Les opportunités et les équipements pour l'apprentissage sont largement disponibles pour aider les apprenants à gérer leur développement 
professionnel continu, et à acquérir des compétences relatives à la résolution de problèmes et la négociation nécessaires aux diplômés dans leur futur rôle de leadership.

4.4 - Les étudiants se voient offrir une exposition précoce et longitudinale à des expériences d'apprentissage basées dans la communauté, à la fois en théorie et en pratique, pour comprendre et agir sur les déterminants de santé et acquérir des habiletés cliniques appropriées. La faculté s'engage pleinement à ce qu'une telle formation soit intégrée dans toutes les disciplines et qu'une utilisation optimale des ressources soit faite au bénéfice de la communauté concernée.

4.5 - La faculté de médecine offre une gamme de services et des mécanismes pour soutenir ses enseignants et ses étudiants à mettre en œuvre des stratégies éducatives et garantit que les diplômés possèdent les compétences attendues qu'exige un système de santé attentif aux préoccupations sociales.

4.6 - La faculté de médecine évalue régulièrement la performance des étudiants dans l'acquisition de l'ensemble des compétences comme décrit dans l'axe 3.

4.7 - Les méthodes et stratégies éducatives sont périodiquement révisées et actualisées en accord avec les bonnes pratiques en matière d'éducation médicale, l'évaluation de la performance des étudiants, les expériences des médecins en pratique et le retour d'information de la part des étudiants et autres parties prenantes du système de santé. De telles révisions s'intéressent particulièrement à vérifier la cohérence entre les valeurs affichées par la faculté, d'une part et les politiques et pratiques observées, d'autre part.

\section{AXE 5. Installation d'une gouvernance réactive et responsable}

5.1 - La faculté de médecine démontre une gouvernance et un leadership responsables pour assumer pleinement son rôle d'acteur clé dans le système de santé et dans la politique de développement des professions de santé, en intégrant les principes de responsabilité sociale dans les programmes d'enseignement, de recherche et de prestation de services.

5.2 - La faculté de médecine engage l'ensemble du corps académique et les étudiants à répondre aux besoins et aux défis de santé dans la société. Un tel engagement est reconnu et fait l'objet d'évaluations conduites de manière systématique et critique, avec des outils certifiés.

5.3 - La faculté de médecine développe des partenariats durables avec d'autres intervenants, telles que les écoles professionnelles de santé, pour optimiser les performances des uns et des autres, notamment en formant des diplômés en qualité et en quantité et en garantissant leur bon déploiement et un meilleur impact sur la santé.

5.4 - La faculté de médecine s'assure que les ressources existantes sont justement distribuées et gérées de manière efficiente et que de nouvelles ressources sont recherchées pour lui permettre de fonctionner en tant qu'institution socialement responsable. Les ressources sont utilisées pour le recrutement de cadres compétents, le bon fonctionnement des infrastructures et la bonne mise en place de nouveaux programmes, en veillant à un juste équilibre entre tous les niveaux de prestation des services de santé.

AXE 6. Redéfinition de normes pour l'éducation, la recherche et la prestation de services

6.1 - L'excellence académique est reconnue comme la capacité d'offrir des programmes d'éducation, de recherche et de prestation de services susceptibles de répondre au mieux aux défis et besoins en santé de la société et d'avoir ainsi un plus grand impact sur la santé. Suivant les principes de responsabilité sociale, l'éventail des normes pour l'évaluation et l'accréditation reflète le continuum de l'identification des problèmes, des choix stratégiques, des processus de gestion, des résultats et de l'impact sur la santé, à la fois sur le plan individuel et populationnel. 
6.2 - Les normes en vigueur dans l'éducation médicale sont revisitées et enrichies avec de nouvelles normes, de sorte que leur éventail comprenne les intrants (qui est formé et par qui), les processus, les résultats (ce que les diplômés réalisent une fois dans la pratique) et l'impact sur la santé. Les normes reflètent le continuum depuis la formation pré-graduée jusqu'à la formation post graduée, en incluant le développement professionnel continu. Les normes en matière de recherche et de prestation de services sont également orientées pour répondre à des besoins de société bien définis et la satisfaction de ces besoins est évaluée et connue des responsables concernés.

6.3 - Les normes relatives aux programmes d'éducation couvrent les champ suivants : la définition des compétences attendues; la coordination avec d'autres professionnels de la santé ; la conception et la révision du curriculum; la coordination et l'aide pour sa mise en œuvre; la formation des enseignants; le recrutement, la sélection, le suivi et le conseil des étudiants; l'allocation des ressources et la gestion de celles-ci; l'évaluation des étudiants, des programmes et des enseignants; la vérification de l'acquisition des compétences attendues pour tous les diplômés; et enfin l'évaluation continue des choix de carrière et de l'engagement professionnel des diplômés à servir dans les zones défavorisées. Les normes sont énoncées et utilisées d'une manière à encourager l'innovation et stimuler la créativité en réponse aux besoins de la société.

6.4 - Les normes relatives aux résultats et à l'impact de l'éducation couvrent les champs suivants : les choix de carrière des diplômés en réponse aux défis et besoins prioritaires de la société en matière de soins de santé; le déploiement et l'affectation de ceux-ci là où ils sont le plus nécessaire; leur capacité à répondre de manière efficiente aux priorités de santé ; l'amélioration de leurs conditions de travail, leur contribution à l'amélioration de l'état de santé de l'ensemble de la population, dans le territoire où la faculté est implantée.

6.5 - Les normes relatives à la gouvernance d'une faculté de médecine couvrent les champs suivants : la qualité de la gouvernance institutionnelle ; la qualité du leadership ; le professionnalisme des cadres académiques ; l'utilisation appropriée des ressources ; la capacité à créer et maintenir des partenariats forts avec les principaux acteurs du système de santé. Tous ces éléments contribuent à l'application pratique des principes de responsabilité sociale.

6.6 - Les normes d'accréditation explorent aussi une formation associant d'autres professionnels de la santé et vérifient les compétences des diplômés à travailler avec d'autres professionnels.

\section{AXE 7. Amélioration continue de la qualité en éducation, recherche et prestation de services}

7.1 - La faculté de médecine s'engage dans un processus d'amélioration et de révision périodique de la qualité, guidé par des normes définies en éducation, en recherche et en prestation de services. L'adhésion à de telles normes est une partie essentielle de la responsabilité sociale des facultés de médecine.

7.2 - La faculté de médecine mesure le progrès en lien avec sa responsabilité sociale par une série de mesures tant qualitatives que quantitatives qui reflètent sa performance au regard de normes valides et fiables relatives aux intrants, processus et résultats. La détermination de ces mesures doit résulter d'un dialogue avec les principaux acteurs de santé, en vue de satisfaire les besoins et défis actuels et futurs en santé.

7.3 - La faculté de médecine soutient fermement l'utilisation systématique et périodique d'outils de mesure pour son évaluation et sa démarche de qualité. Le processus est explicite, transparent, constructif et accessible aux différents acteurs de santé.

7.4 - Dans sa démarche de qualité et pour prétendre devenir socialement responsable, la faculté de médecine reconnaît le rôle déterminant d'une structure de gouvernance performante, d'un leadership responsable, ainsi que de normes professionnelles pour le personnel engagé dans les domaines de l'éducation médicale, de la recherche et des prestations de services. 


\section{AXE 8. Institutionalisation de mécanismes d'accréditation}

8.1 - L'accréditation est un puissant levier pour le changement institutionnel et l'amélioration de la qualité. Elle doit être activement encouragée par les autorités académiques et sanitaires à travers le monde. L'existence d'un mécanisme dans le pays ou la région permet à toute faculté de médecine d'être accréditée par un organisme reconnu. Une accréditation est effectuée à intervalles réguliers conduisant entre-temps à des améliorations notables.

8.2 - Les normes et processus d'accréditation reflètent les principes de responsabilité sociale en autant qu'ils couvrent l'état des intrants, processus, résultats et l'impact, permettant d'apprécier et de promouvoir la capacité des facultés de médecine à répondre de façon efficiente aux défis et aux besoins en santé de la société.

8.3 - L'existence d'un mécanisme d'accréditation implique aussi l'existence d'un soutien aux facultés de médecine dans leur effort à se conformer aux normes et au processus. Selon le contexte, ce soutien varie et peut aller de l'énoncé de directives favorisant la responsabilité sociale à la mise à disposition de moyens ou autres incitatifs.

8.4 - L'évaluation interne est confirmée par une évaluation externe conduite par des pairs. Des représentants des acteurs de santé sont invités à participer à la définition des normes, à la sélection des évaluateurs externes et à une mise à jour régulière du système d'accréditation.

\section{AXE 9. Adhésion aux principes universels et adaptation au contexte local}

9.1 - Les principes de responsabilité sociale sont universels : ils doivent être adoptés et appliqués à travers le monde, car ils tendent à améliorer la capacité d'une faculté de médecine à mieux utiliser son potentiel pour identifier et relever les défis de la santé et les besoins de la société dans un esprit de qualité, d'équité, de pertinence, d'innovation et d'utilisation appropriée des ressources.

9.2 - Vu la mobilité internationale accrue des médecins, mais aussi des patients, la faculté de médecine est consciente des phénomènes de mondialisation. Dans le cadre du développement durable au niveau mondial, elle doit introduire des perspectives internationales dans la conception, l'organisation et la mise en œuvre de ses enseignements.

9.3 - Bien que les principes, définitions et classifications en matière de responsabilité sociale soient universels, leur adaptation au contexte local est primordiale.

9.4 - Les organisations internationales actives dans les domaines de la santé et de l'enseignement supérieur, au niveau régional ou mondial, doivent préconiser les systèmes d'assurance qualité, tels que l'accréditation. Un cadre de régulation est nécessaire pour que soient appliqués les principes de la responsabilité sociale afin de mieux répondre aux besoins de santé prioritaires des pays et faire face à la crise générale des ressources humaines dans le secteur de la santé.

\section{AXE 10. Prise en compte du rôle de la société}

10.1 - Il y a un équilibre à trouver entre la préservation de l'autonomie institutionnelle et la place des différents acteurs de santé y compris de la société civile pour intégrer les principes de responsabilité sociale dans les facultés de médecine. Il s'agit là d'un véritable défi.

10.2 - Les principaux acteurs, à savoir, les décideurs politiques, les gestionnaires d'organisations de santé, les professionnels de la santé et la société civile, sont représentés dans les équipes d'évaluation interne et externe, y compris pour l'accréditation, car la responsabilité sociale concerne directement ces acteurs.

10.3 - Les communautés où la faculté de médecine est implantée sont régulièrement consultées pour obtenir leur avis sur le niveau de responsabilité sociale de la faculté. Les résultats et les observations de l'accréditation de la faculté sont rendus publics. 


\title{
Annexes
}

\section{Comité de pilotage}

Magdalena Awases (Human Resources for Health - HRH -, World Health Organzation - WHO -

Regional Office for Africa)

Rebecca Bailey* (HRH, WHO Geneva)

Charles Boelen, co-chair* (Formerly with HRH, WHO)

Mario Dal Poz HRH (WHO Geneva)

Moses Galukande* (Makerere University and International Medical Group)

Jorge Eduardo Gutiérrez Calivá (Asociación Castarricense de Facultades y Escuelas de

Medicina - ACOFEMED)

Dan Hunt (Liaison Committee on Medical Education - LCME)

Jehu Iputo* (Walter Sisulu University)

Yusuf Irawan (Hasanuddin University)

Ahmed Kafajei Jordan (University of Sciences and Technology)

Joël Ladner* (Université de Rouen)

Sam Leinster* (University of East Anglia)

Stefan Lindgren* (World Federation for Medical Education - WFME - and Lund University)

Khaya Mfenyana* (Walter Sisulu University)

Tewfik Nawar* (Université de Sherbrooke)

Andre-Jacques Neusy* (Training for Health Equity Network - THEnet)

Björg Pálsdóttir* (THEnet)

Jean Rochon (Université Laval)

Robert Woollard, co-chair* (University of British Columbia)

\section{Groupe de référence international}

\author{
Mohamed Elhassan Abdalla* (Jazan University) \\ Ibrahim Abdulmeini (Registrar of the Medical and Dental Councils of Nigeria) \\ Walid Abubaker* (WHO/EMRO) \\ Liliana Arias (Columbian Association of Medical Schools, Member of Admin Committee at PAFAMS) \\ Carol Aschenbrener (American Association of Medical Colleges - AAMC) \\ Makonnen Asefa* (Ethiopian Public Health Association/World Federation of Public Health \\ Associations - WFPHA) \\ Sébastian Audette (Accreditation Canada) \\ Djona Avoksouma (WHO Regional Office for Africa) \\ Ibrahim Banihani (Association of Medical Schools in the Eastern Mediterranean Region) \\ Rashad Barsoum (Supreme Council of Egyptian Universities) \\ Barbara Barzansky (LCME) \\ Mourad Belaciano (Asociación Brasileña de Educación Médica-ABEM) \\ Dan Benor (Ben Gurion University) \\ John Bligh (Academy of Medical Educators)
}


Dan Blumenthal* (Morehouse School of Medicine)

Bettina Borisch (WFPHA)

Rosa Maria Borrell-Bentz (WHO America Office)

Nick Busing (Association of Faculties of Medicine of Canada AFMC)

David Buso* (Walter Sisulu University)

Jim Campbell

Emmanuel Cassimatis (Educational Commission for Foreign Medical Graduates - ECFMG)

Francesca Celetti (HRH, WHO Geneva)

Angel Centeno* (Austral University)

Lincoln Chen (China Medical Board)

Shakuntala Chhabra* (MG Institute of Medical Science - MGIMS)

Ian Couper* (University of Witwatersrand)

Manuel Dayrit (HRH, WHO Geneva)

Milton de Arruda Martins (University of Sao Paulo)

Eric de Roodenbeke (International Hospital Federation - IHF-FIH)

Jean-Francois Denef* (International French Speaking Society for Medical Education - SIFEM)

Horacio Deza (Association of Medical Schools of the Argentine Republic AF-ACIMERA)

Mohenou Isidore Jean-Marie Diomande (UFR Sciences Médicales d'Abidjan)

Robbert Duvivier (International Federation of Medical Student Associations - IFMSA)

Tetanye Ekoe* (Yaounde Faculty of Medicine)

Amal Elouazzani* (Hassan II University)

Mohamed Elsanousi* (University of Gezira)

Julian Fisher (FDI World Dental Federation)

Antoine Flahault (Association of Schools of Public Health in the European Region - ASPHER)

Cristobal Fortunato (Ateneo de Zaboanga University)

Jean-Paul Francke (International Association of Deans of Francophone Medical Schools - CIDMEF)

Tabeh Freeman (Liberia Medical School)

Seble Frehywot (George Washington University)

George Gage (Afro European Medical and Research Network)

Jacob Gayle (The Ford Foundation)

Grace George* (Walter Sisulu University)

Trevor Gibbs

Charles Godue (HRF, WHO Regional Office for the Americas/PAHO)

Ioana Goganau* (International Federation of Medical Students Associations - IFMSA)

Rajesh Gongal* (Patan Academy of Health Sciences, Patan Hospital)

David Gordon* (Association of Medical Schools in Europe - AMSE)

Arcadi Gual (Fundacion Educacion Medica)

Edward Gyader* (School of Medicine and Health Sciences, Ghana)

Georges Haddad (UNESCO)

John Hamilton* (University of Newcastle)

Bashir Hamad* (University of Gezira)

Jason Hilliard (University of Colorado)

Yiqun Hu (Shanghai Jiaotong University)

Manuel Huaman (Asociación Peruana de Facultades de Medicina-ASPEFAM) 
Muzaherul Huq (HRH, WHO Regional Office for South-East Asia)

Yojiro Ishii (Japan International Cooperation Agency)

Marian Eslie Jacobs* (Association of Medical Schools in Africa - AMSA - and University of Cape Town)

Susan Johnson* (National Board of Medical Examiners - NBME - and University of Iowa)

SAS Kargbo (Ministry of Health, Sierra Leone)

Geoffrey Kasembeli (Afro European Medical and Research Network)

Zulfiqar Khan* (WHO Pakistan)

Michael Kidd (WONCA working party on Education)

Khunying Kobchitt Limpaphayom (South East Asian Regional Association on Medical Education)

Joseph Kolars (Bill and Melinda Gates Foundation)

Jan Maeseneer (The Network Towards Unity for Health-Network TUFH)

Henri Manasse Jr.* (American Society of Health-System Pharmacists)

Dianne Manning* (University of Witwatersrand)

Maurice McGregor* (McGill University)

Jim McKillop (General Medical Council - GMC - Undergraduate Board)

Donald Melnick (NBME)

Hugo Mercer (Former HRH, WHO)

Mwapatsa Mipando Nader* (University of Malawi)

Momtazmanesh Gottlieb* (Ministry of Health and Medical Education)

Monekosso Fitzhugh Mullan* (Global Health Dialogue)

Richard Murray* (George Washington University)

Jeremiah Mwangi (Australia International Alliance of Patients' Organizations)

Rose Chalo Nabirye* (WFPHA, Uganda)

Sophon Napathorn* (Chulalongkorn University)

Lois Nora* (North-eastern Ohio Universities and Colleges of Medicine and Pharmacy and ICME)

John Norcini* (Foundation for Advancement of International Medical Education and ResearchFAIMER)

Jesus Noyola (Asociación Mexicana de Facultades de Medicina-AMFEM)

Ezekiel Nukuro (HRH, WHO Regional Office for the Western Pacific)

Chacha Nyaigotti-Chacha (Inter-University Council for East Africa-IUCEA)

Francis Omaswa (African Center for Global Health and Social Transformation)

Alberto Oriol Bosch (Fundacion Educacion Medica)

Martins Ovberedjo* (WHO Tanzania)

Neil Pakenham-Walsh (Global Healthcare Information Network)

Jorgi Pales (Sociedad Espanola de Educacion Medica - SEDEM)

Madalena Patricio* (Association for Medical Education in Europe - AMEE)

Galina Perfilieva (Country Policies, Systems and Services, WHO Regional Office for Europe)

Dominique Pestiaux (SIFEM)

David Prideaux* (Flinders University)

Pablo Pulido (Pan American Federation of Associations of Medical Schools - PAFAMS/FEPAFEM)

Rich Roberts (WONCA President elect)

Paschalis Rugarabam* (Hurbet Kairuki Memorial University)

Mubashar Sheikh (Global Health Workforce Alliance - GHWA)

Jusie Siega-Sur* (University of the Philippines) 
Leslie Southgate (Academy of Medical Educators)

David Stern (Institute for International Medical Education)

Roger Strasser (Northern Ontario School of Medicine)

Navin Sunderlall* (University of Kwazulu Natal)

Kate Tulenko (IntraHealth International)

Felix Vartanian* (Russian Academy of Advanced Medical Studies)

Anvar Velji* (Global Health Education Consortium)

Kuku Voyi (University of Pretoria)

Margot Weggemans (IFMSA)

Gustaaf Wolvaardt* (Foundation for Professional Development)

Liz Wolvaardt* (University of Pretoria)

Paul Worley (Flinders University)

Akemi Yonemura (UNESCO)

Toshimasa Yoshioka (Association of Medical Schools in the Western Pacific Region)

* Membres ayant assisté à la conférence pour le consensus mondial sur la responsabilité sociale à East London (Afrique du Sud), du 10 au 13 octobre 2010. 